\title{
X-Ray Microanalysis with High Spatial Resolution and High Counts Rate with a State of the Art Field Emission Scanning Electron Microscope
}

\author{
Raynald Gauvin ${ }^{1}$, Nicolas Brodusch ${ }^{1}$, Hendrix Demers ${ }^{1}$ and Patrick Woo ${ }^{2}$ \\ 1. Department of Mining and Materials Engineering, McGill University, Montreal, Quebec, Canada. \\ 2. Hitachi High-Technologies Canada Inc., Toronto, Canada.
}

The scanning electron microscope (SEM) was primary developed for imaging applications. With the introduction of the $\mathrm{Si}(\mathrm{Li})$ energy dispersive spectrometer (EDS), simultaneous imaging and x-ray microanalysis became possible. However, long working distance and high current were needed because the position and small solid angle of the EDS detector. SEM was initially and is still optimized for imaging applications, where the high spatial resolution is generally obtained at short working distance. This problem is still relevant today and unfortunately x-ray microanalysis is never performed in the best imaging conditions, i.e., not with the smallest probe size. With the introduction of an annular silicon drift detector (SDD) system, scanning electron microscopy is facing a revolution. This detector is inserted below the objective lens which gives a higher solid angle (up to $1.2 \mathrm{sr}$ ). Also, a lower working distance and probe current can be used. An improved spatial resolution becomes possible during x-ray microanalysis. At this point, the time required for $\mathrm{x}$-ray imaging will be of the same order as for the atomic number contrast images achieved through backscattered electrons (BSE) imaging [1].

Carbon nanotubes (CNTs) decorated with nanoparticles are often used to evaluate the high spatial resolution of state of the art FE-SEM. Figure 1 shows an example of high spatial resolution imaging of CNTs. The dark-field micrograph had a spatial resolution of $6.5 \mathrm{~nm}$, which was calculated with SMART-J [2-3]. However, the accelerating voltage and working distance were not optimum for this kind of imaging. They were chosen to allow simultaneously the acquisition of x-ray map with an annular (SDD). Currently, this system is limited to accelerating voltage below $20 \mathrm{kV}$ and the shortest working distance is around $10 \mathrm{~mm}$, which is shorter than the one used with a conventional SDD (15 mm on our system). Figure 2 shows a platinum x-ray map obtained with an annular SDD. A probe current of $270 \mathrm{pA}$ (count rate of $7 \mathrm{kcps}$ ) and an acquisition time of 7 minutes were used. A spatial resolution of 8.9 nm was calculated for this X-ray "micrograph".

With this $x$-ray detector installed on a cold field emission scanning electron microscope, like the HITACHI SU-8230, quantitative x-ray microanalysis with high spatial resolution at low beam energy and low current becomes possible with the possibility of using the various different type of imaging at the same time. Also, since the count rate can be as high as 1,500 kcps with our system, which lower significantly the detection limit of elements as well the minimum feature sizes of different phases that can be distinguished.

\section{Reference:}

[1] D.E. Newbury and N.W.M. Ritchie, Scanning, 33 (2011), pp. 174-192.

[2] D.C. Joy, Journal of Microscopy, 208 (2002), pp. 24-34.

[3] J. Kim et al, Journal of Vacuum Science Technology B, 25 (2007), pp. 1771-1775. 


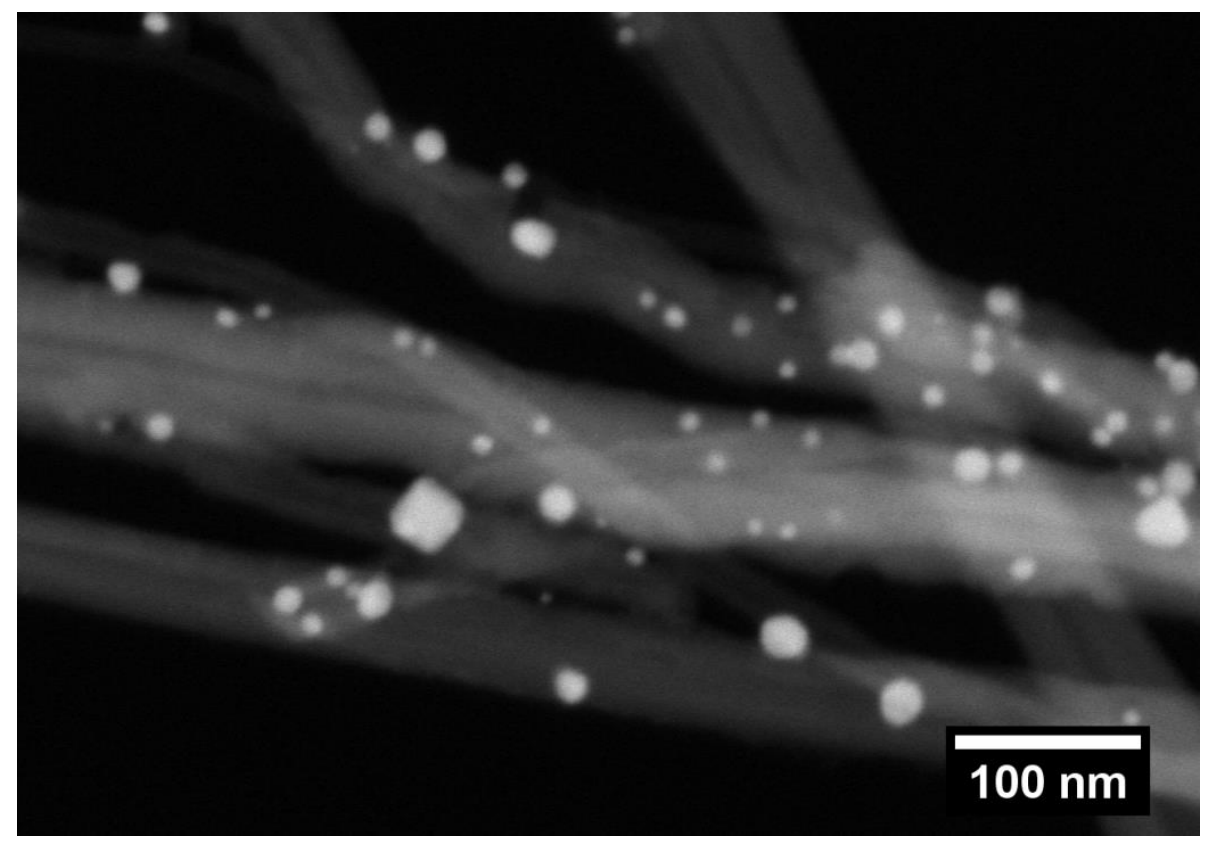

Figure 1. Dark field micrograph of carbon nanotubes decorated with platinum $(\mathrm{Pt})$ nanoparticles was acquired in a low voltage scanning transmitted electron microscope. A resolution of $6.5 \mathrm{~nm}$ was measured with SMART-J [2-3]. An accelerating voltage of $20 \mathrm{kV}$ and a working distance of $10.5 \mathrm{~mm}$ were used.

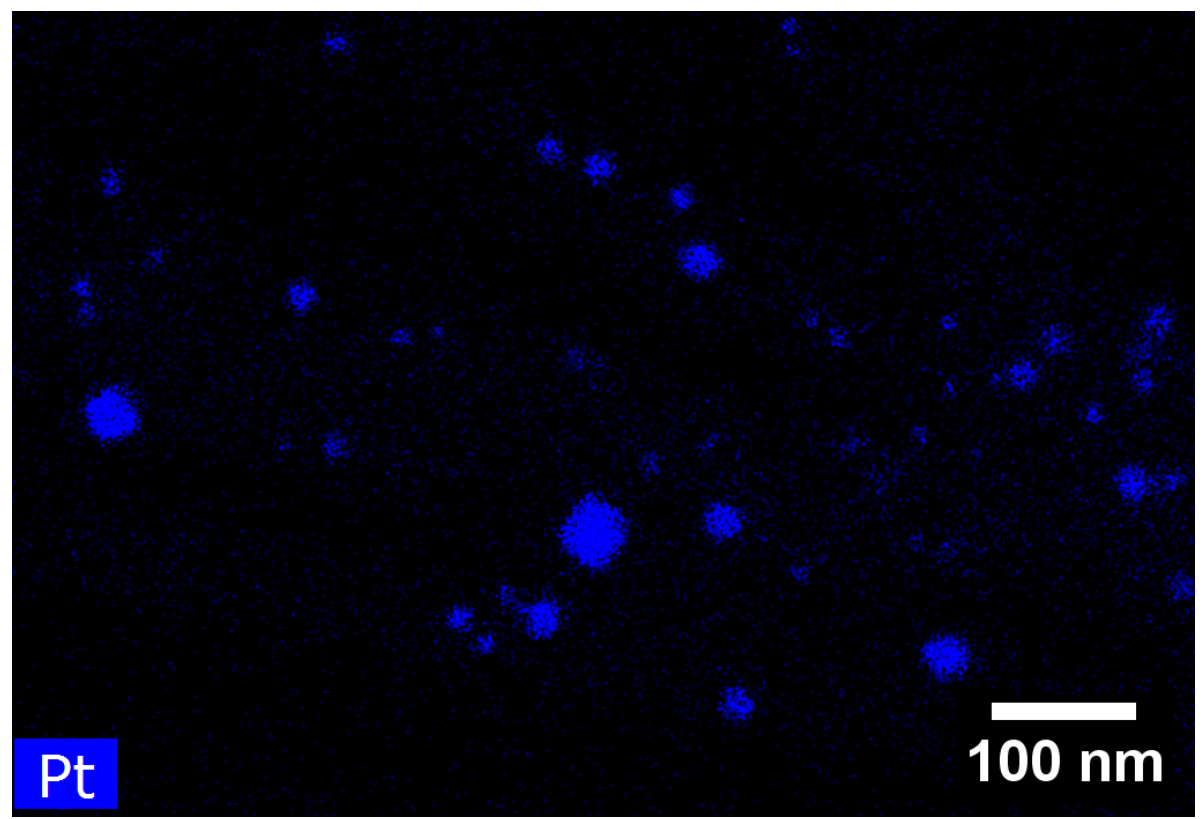

Figure 2. X-ray map of carbon nanotubes decorated with platinum (Pt) nanoparticles was acquired with an annular Silicon drift detector. A resolution of $8.9 \mathrm{~nm}$ was measured with SMART-J [2-3]. The map acquisition time was $412 \mathrm{~s}$ with a count rate of $7 \mathrm{kcps}$. An accelerating voltage of $20 \mathrm{kV}$ and a working distance of $10.5 \mathrm{~mm}$ were used. 